\title{
Electrochemical Capacitors Based on Aligned Carbon Nanotubes Directly Synthesized on Tantalum Substrates
}

\author{
Byungwoo Kim, Haegeun Chung, Byoung Koun Min, ${ }^{\dagger}$ Honggon Kim, ${ }^{\dagger}$ and Woong Kim* \\ Department of Materials Science and Engineering, Korea University, Seoul 136-713, Korea \\ ${ }^{*}$ E-mail: woongkim@korea.ac.kr \\ ${ }^{\dagger}$ Clean Energy Center and ${ }^{\star}$ Solar Cell Center, Korea Institute of Science and Technology, Seoul 136-791, Korea \\ Received October 1, 2010, Accepted October 11, 2010
}

\begin{abstract}
We demonstrate that vertically aligned carbon nanotubes can be synthesized directly on tantalum substrate via waterassisted chemical vapor deposition and evaluate their properties as electrochemical capacitors. The mean diameter of the carbon nanotubes was $7.1 \pm 1.5 \mathrm{~nm}$, and $70 \%$ of them had double walls. The intensity ratio of G-band to D-band in Raman spectra was as high as 5 , indicating good quality of the carbon nanotubes. Owing to the alignment and low equivalent series resistance, the carbon nanotube based supercapacitors showed good rate performance. Rectangular shape of cyclic voltammogram was maintained even at the scan rate of $>1 \mathrm{~V} / \mathrm{s}$ in $1 \mathrm{M}$ sulfuric acid aqueous solution. Specific capacitance was well-retained ( $94 \%)$ even when the discharging current density dramatically increased up to $145 \mathrm{~A} / \mathrm{g}$. Consequently, specific power as high as $60 \mathrm{~kW} / \mathrm{kg}$ was obtained from as-grown carbon nanotubes in aqueous solution. Maximum specific energy of $\sim 20 \mathrm{Wh} / \mathrm{kg}$ was obtained when carbon nanotubes were electrochemically oxidized and operated in organic solution. Demonstration of direct synthesis of carbon nanotubes on tantalum current collectors and their applications as supercapacitors could be an invaluable basis for fabrication of high performance carbon nanotube supercapacitors.
\end{abstract}

Key Words: Carbon nanotubes, Chemical vapor deposition, Tantalum, Supercapacitors

\section{Introduction}

Electrochemical capacitors, so called supercapacitors or ultracapacitors, are promising energy storage devices that may replace or complement batteries. ${ }^{1-4}$ In general, supercapacitors have high power density, long cycle life, and are made of environmentally friendly materials. Currently, porous activated carbon is one of the most commonly used materials due to its high specific surface area. ${ }^{1,5}$ However, the activated carbon has irregular pore structures that can reduce ion diffusion rate. ${ }^{6,7}$ Moreover, binding agent is required to attach the activated carbon to current collectors, which can increase equivalent series resistance (ESR). ${ }^{7}$ Therefore, performance enhancement can be expected by replacing the porous activated carbon with other materials that have regular pore structures and by developing binder-free fabrication processes.

Carbon nanotubes (CNTs) are good candidate materials to replace the activated carbon because they have excellent properties such as high conductivity, low mass density, high mechanical strength, large specific surface area, chemical stability, regular pore structures, and continuous conductive paths. ${ }^{8-11}$ Moreover, integration of CNTs with current collectors without using binder agents is possible by synthesizing CNTs directly on current collector substrates. While aligned CNTs have been grown mostly on insulating substrates, ${ }^{12-15}$ it has been recently demonstrated that they can be synthesized directly on conductive substrates without using any insulating layers via vaporphase catalyst delivery. ${ }^{16}$ However, this process is relatively complicated and requires a separate heating chamber to vaporize carbon and catalyst precursors. Very recently, our group successfully synthesized vertically aligned CNTs directly on conductive substrates such as stainless steel and carbon papers via a simple water-assisted chemical vapor deposition (CVD). ${ }^{17,18}$

In this paper, we demonstrate that our direct CNT synthesis can be extended to tantalum (Ta) substrates and evaluate CNT properties for electrochemical capacitor applications. Vertically aligned CNTs with a mean diameter of $\sim 7 \mathrm{~nm}$ were obtained. Approximately $70 \%$ of them were double walled CNTs. The CNT supercapacitors showed excellent rate performance which was attributed to the good alignment of the CNTs and low $\mathrm{ESR}^{6,7}$ Almost ideal rectangular CV shape was observed at the scan rate of $\sim 1 \mathrm{~V} / \mathrm{s}$, and specific capacitance was retained $(94 \%$ retention) even at a high discharge current density of $145 \mathrm{~A} / \mathrm{g}$. Maximum specific power of the as-grown CNTs was approximately $60 \mathrm{~kW} / \mathrm{kg}$. Electrochemical oxidation of the CNTs and operation of the supercapacitors with them in organic solution improved the specific energy by introducing pseudocapacitance, increasing the effective surface area of CNTs, and widening the operation voltage range. ${ }^{1,18,19}$ Maximum specific energy of 20 $\mathrm{Wh} / \mathrm{kg}$ was obtained from oxidized CNTs. Demonstration of direct integration of CNTs with current collectors could be an important basis for development of high performance energy storage devices.

\section{Experimental Section}

Growth and characterization of CNTs. Ta (Sigma Aldrich, 99.9\%) substrates were cleaned by sonication in de-ionized (DI) water, in acetone and in ethanol for $5 \mathrm{~min}$, respectively. To synthesize CNTs on the Ta substrates, $10 \mathrm{~nm}$ of aluminum (Al) and $1 \mathrm{~nm}$ of iron $(\mathrm{Fe})$ layer were deposited by e-beam evaporation. The substrates were placed in the middle of quartz tube in 
a furnace. While the furnace was heated, hydrogen $(99.999 \%)$ and $\operatorname{Ar}(99.999 \%)$ were introduced at flow rates of 100 and 125 sccm, respectively. Small amount of water vapor was introduced simultaneously by passing Ar through a DI-water bubbler at $0.75 \mathrm{sccm}$. Once the temperature reached $800{ }^{\circ} \mathrm{C}$, ethylene $(99.95 \%)$ started to flow at $50 \mathrm{sccm}$ as a carbon source. After typical 5 min of reaction time, the furnace was cooled down in the flow of Ar and samples were taken from the quartz tube near room temperature. Morphology and fine structure of CNTs were characterized with scanning electron microscopy (SEM, FEI Nova nano 200) and transmission electron microscopy (TEM, Philips Tecnai 20). Quality of the CNTs was evaluated with Raman spectroscopy (Horiba, HR 800).

Measurement of supercapacitor properties. Cyclic voltammetry $(\mathrm{CV})$, galvanostatic charge-discharge measurement, and electrochemical impedance spectroscopy (EIS) were carried out in a conventional three-electrode system using an electrochemistry analyzer (Ivium Technologies, CompactStat). CNTs on a Ta substrate, a platinum gauze (Sigma-Aldrich, 99.9\%), and an $\mathrm{Ag} / \mathrm{AgCl}$ electrode (CH Instruments, $\mathrm{CHI} 111,3 \mathrm{M} \mathrm{KCl}$ ) were used as a working, a counter and a reference electrode, respectively in $1 \mathrm{M}$ sulfuric acid $\left(\mathrm{H}_{2} \mathrm{SO}_{4}\right)$ aqueous solution. In $1 \mathrm{M}$ tetraethylammonium tetrafluoroborate $\left(\mathrm{Et}_{4} \mathrm{NBF}_{4}\right)$ propylene

(a)

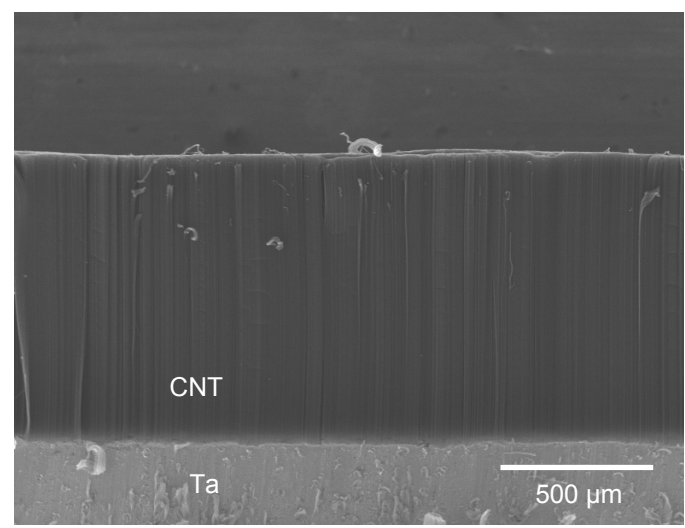

(c)

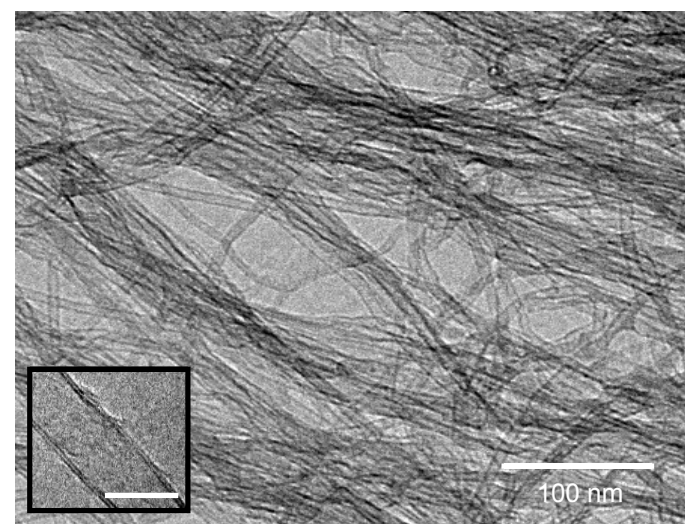

carbonate (PC) solution, a Ag wire (99.99\%) was used instead of the $\mathrm{Ag} / \mathrm{AgCl}$ reference electrode. Potential ranges of $\mathrm{CV}$ and galvanostatic charge-discharge current measurement were $0 \sim 1 \mathrm{~V}$ and $-1.25 \sim 1.25 \mathrm{~V}$ in aqueous and organic solution, respectively. Frequency range of the EIS was $100 \mathrm{KHz} \sim 0.1 \mathrm{~Hz}$. Electrochemical oxidation of CNTs was carried out in $0.25 \mathrm{M}$ nitric acid $\left(\mathrm{HNO}_{3}\right)$ aqueous solution. The potential was scanned in the range of 1.0 to $2.0 \mathrm{~V}$ at $50 \mathrm{mV} / \mathrm{s}$ sweep rate for 10 cycles. Specific energy and specific power was calculated based on the following equations: $E_{\mathrm{sp}}=1 / 2 \times C_{\mathrm{sp}} V^{2}$ and $P_{\mathrm{sp}}=E_{\mathrm{sp}} / t$, where $C_{\text {sp }}$ is specific capacitance estimated from a slope of a discharge curve $(\mathrm{d} V / \mathrm{d} t)$ after IR drop, and $V$ and $t$ are a potential and a time component of the discharge slope, respectively.

\section{Results and Discussion}

A dense CNT film was grown directly on Ta substrates via water-assisted CVD as shown in Figure 1a. Small amount of water added during the synthesis extends catalyst life time by counteracting the deposition of unwanted carbon species on catalyst surface. ${ }^{20}$ Consequently, growth rate was revealed as fast as $\sim 160 \mu \mathrm{m} / \mathrm{min}$. Figure 1a shows approximately $800 \mu \mathrm{m}$ long CNTs grown on a Ta substrate. $\mathrm{Al} / \mathrm{Fe}$ bilayer was used as a

(b)

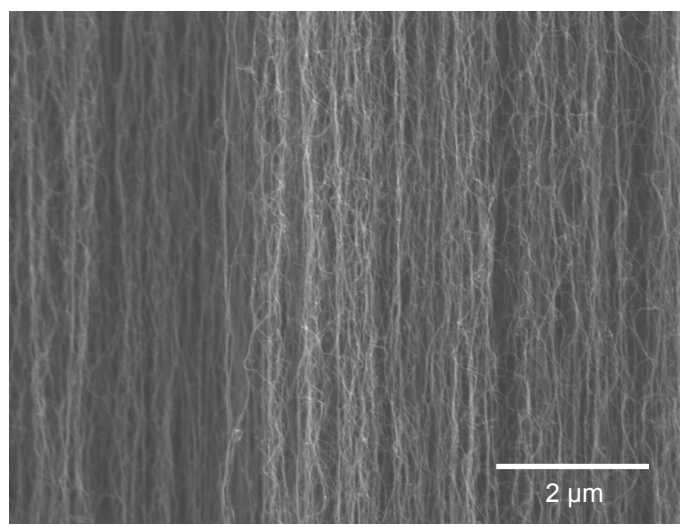

(d)

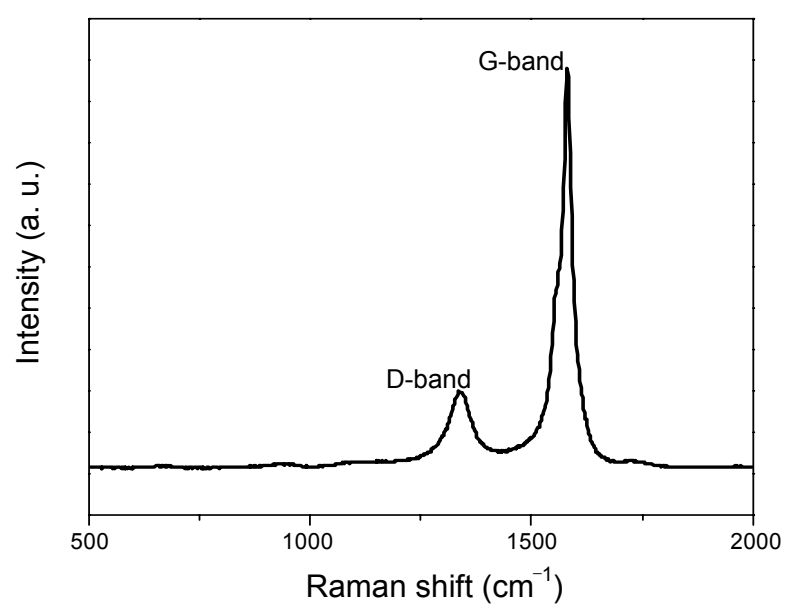

Figure 1. (a) A scanning electron microscope (SEM) image of carbon nanotubes (CNTs) directly synthesized on a tantalum (Ta) plate. (b) An SEM image showing the alignment of CNTs. (c) A transmission electron microscope (TEM) image of the CNTs. Inset shows a double walled CNT (scale bar $=10 \mathrm{~nm})$. (d) A Raman spectrum of the CNTs. 
(a)

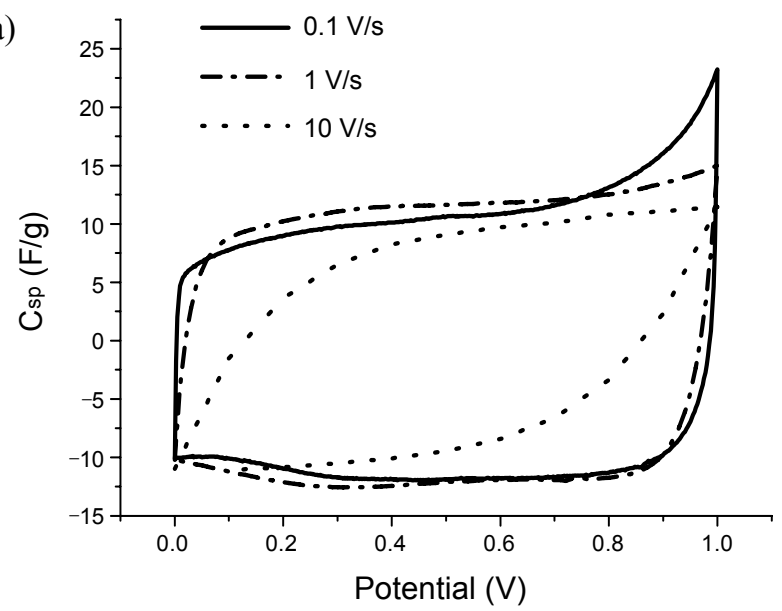

(c)

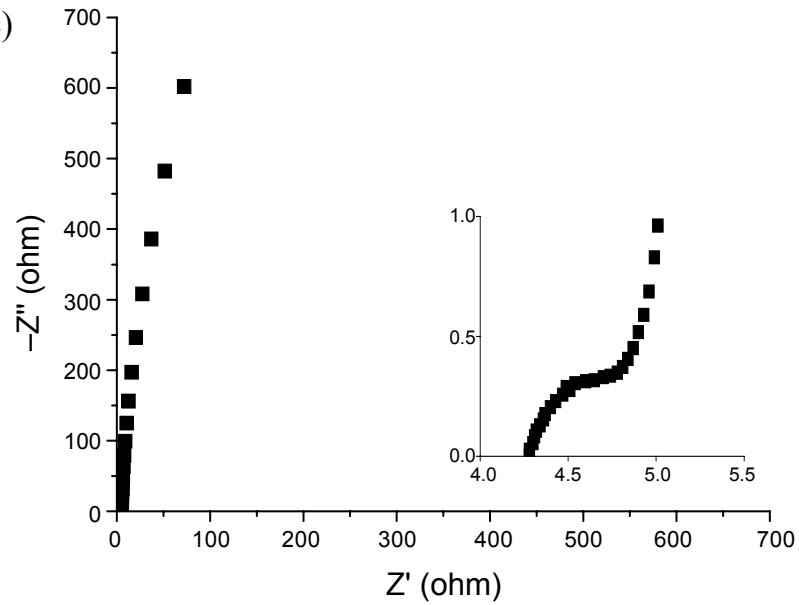

(b)

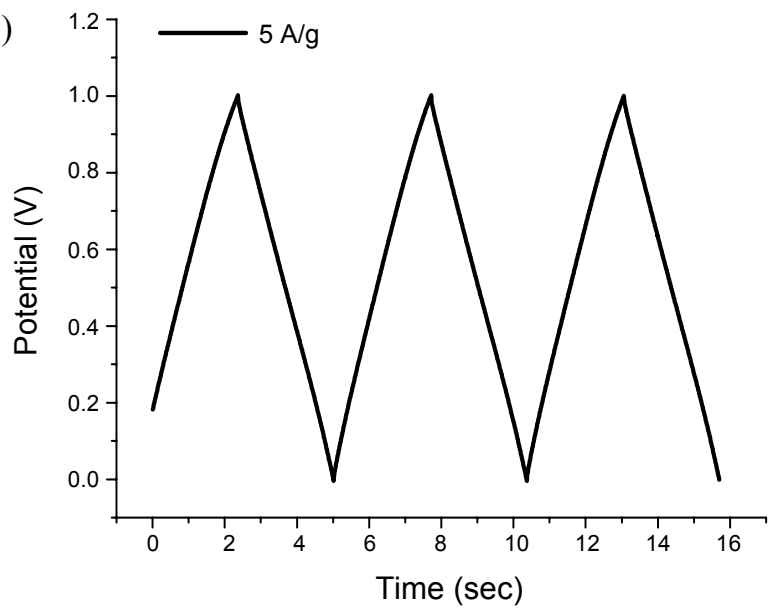

(d)

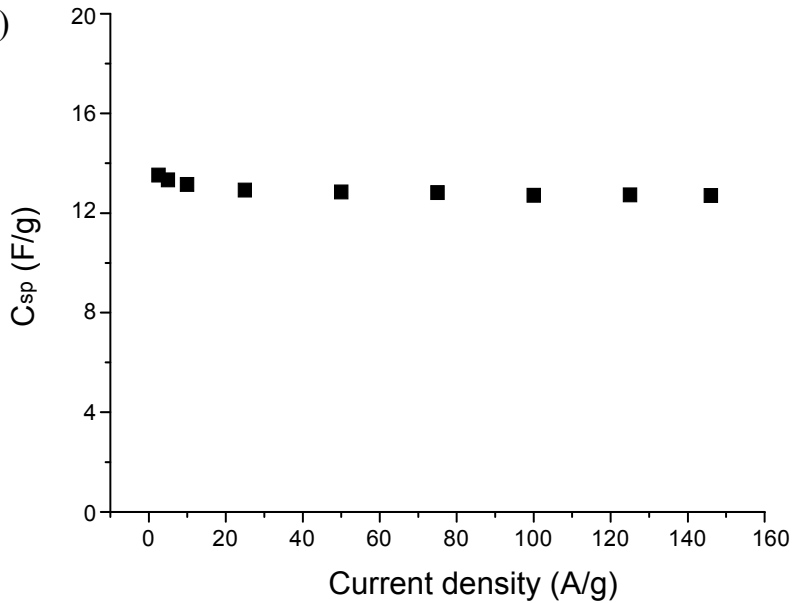

Figure 2. (a) Cyclic voltammogram of as-grown CNTs measured in $1 \mathrm{M} \mathrm{H}_{2} \mathrm{SO}_{4}$ aqueous solution. (b) Galvanostatic charge/discharge curves. (c) Nyquist plot. Inset shows a zoomed-in plot. (d) A plot of specific capacitance vs. current density.

catalyst layer to preferentially produce few-walled CNTs $(d<$ $10 \mathrm{~nm})$ over large multiwalled CNTs $(d<100 \mathrm{~nm})$, since the $\mathrm{Al}$ layer supposedly prevents aggregation of $\mathrm{Fe}$ nanoparticles. ${ }^{21}$ The CNTs were vertically aligned to the substrates, and the alignment was clearly observed with SEM as shown in Figure 1b. TEM characterizations revealed that CNT diameter was $7.1 \pm 1.5 \mathrm{~nm}$ (mean \pm one standard deviation) and that the majority of CNTs ( $\sim 67 \%, 24$ out of 36 CNTs) had double walls (Figure 1c and inset). Quality of the CNTs was decent as confirmed by high G-band to D-band intensity ratio $\left(\mathrm{I}_{\mathrm{G}} / \mathrm{I}_{\mathrm{D}}<5\right)$ of Raman spectra (Figure 1d).

The as-grown CNTs on Ta showed excellent rate performance as electrochemical capacitors. CV curves measured at different scan rates are shown in Figure 2a. Almost ideal rectangular shapes were observed at 0.1 and $1 \mathrm{~V} / \mathrm{s}$, indicating rapid current response to the change of voltage sweep direction. The $\mathrm{CV}$ shape was retained to some degree even at extremely fast scan rate $(\sim 10 \mathrm{~V} / \mathrm{s})$. Specific capacitance estimated from the $\mathrm{CV}$ curves was $\sim 13 \mathrm{~F} / \mathrm{g}$. Galvanostatic charge/discharge curve measured at the current density of $5 \mathrm{~A} / \mathrm{g}$ is shown in Figure 2b. Small IR drop indicates that the ESR of the supercapacitor is negligible. EIS consistently shows the low $\operatorname{ESR}(\sim 4.3 \Omega)$ as shown in a
Nyquist plot (Figure 2c and inset). Finally, the specific capacitance was measured from the galvanostatic charge/discharge curves at various current densities (Figure 2d). Only slight reduction of the specific capacitance was observed, even when the current density was increased up to $145 \mathrm{~A} / \mathrm{g}$. Retention of specific capacitance even at fast scan rate and at high discharge current density are desirable characteristics for high power supercapacitors. This excellent rate performance of CNTs arises mainly from the alignment of the CNTs and direct integration between the CNTs and Ta current collectors, which ensure fast ion diffusion and low ESR of the supercapacitors, respectively. ${ }^{6,13,22}$ Maximum specific power of the as-grown CNTs was estimated to be $\sim 60 \mathrm{~kW} / \mathrm{kg}$.

The specific capacitance and the specific energy of the CNT supercapacitors were increased by electrochemical oxidation of CNTs. Pseudocapacitance can be induced due to oxygenated groups on the CNTs produced by this treatment. ${ }^{18,19}$ Moreover, inner surface of the CNTs become available for ion adsorption because the tips of the CNTs can be opened via the electrochemical oxidation process. ${ }^{18,19}$ The specific capacitance was increased to $\sim 40 \mathrm{~F} / \mathrm{g}$ owing to the additional pseudocapacitance and the increased effective surface area. Maximum specific 
(a)

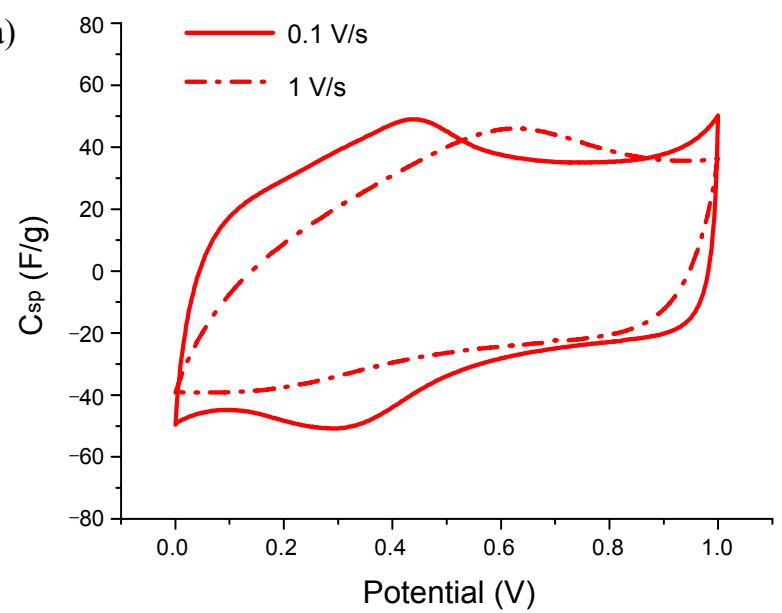

(c)

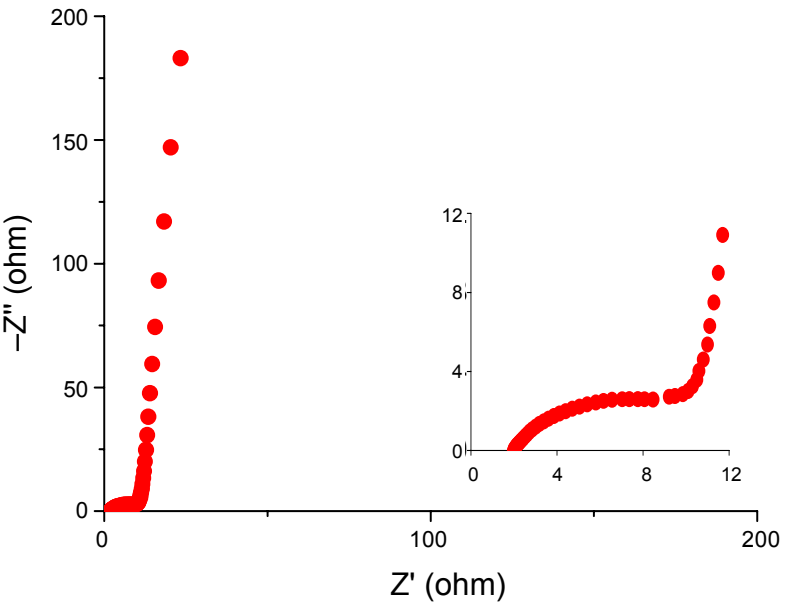

(b)

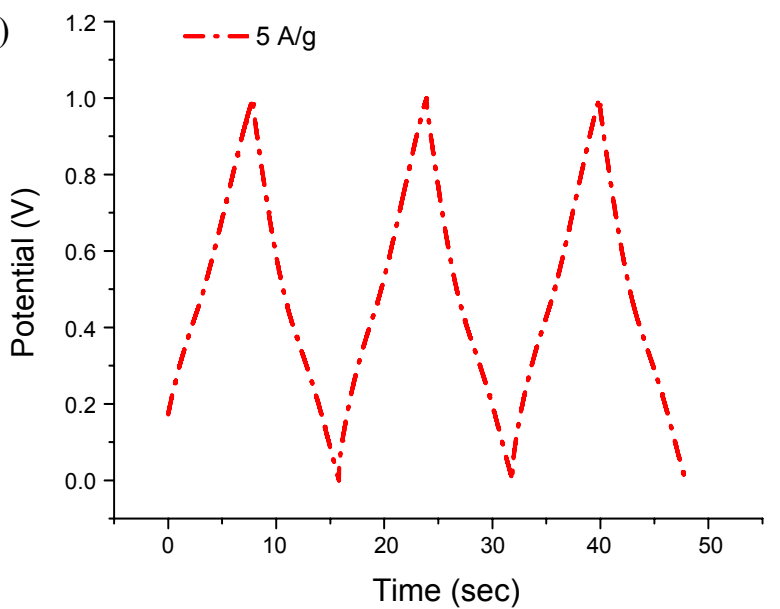

(d)

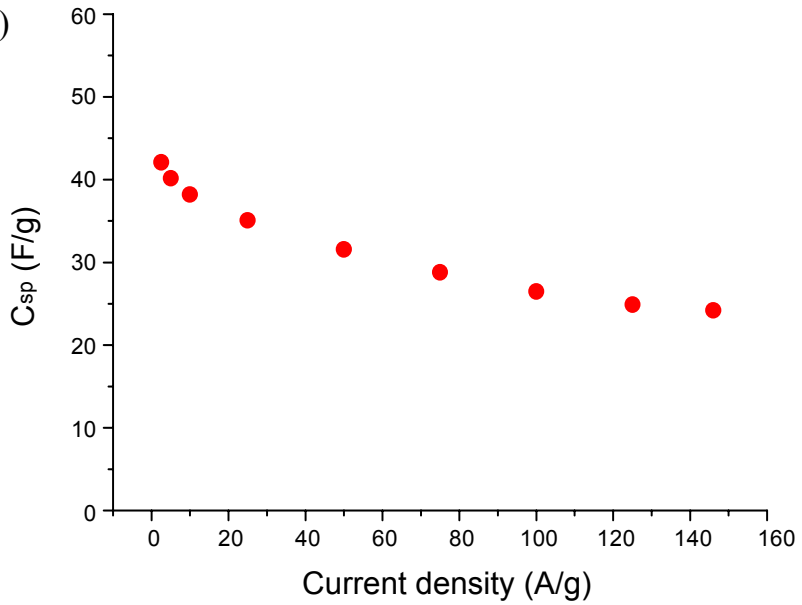

Figure 3. (a) Cyclic voltammogram of electrochemically oxidized CNTs measured in $1 \mathrm{M} \mathrm{H}_{2} \mathrm{SO}_{4}$ aqueous solution. (b) Galvanostatic charge/ discharge curves. (c) Nyquist plot. Inset shows an enlarged plot. (d) A plot of specific capacitance vs. current density.

energy was increased from 2 to $6 \mathrm{Wh} / \mathrm{kg}$ via the electrochemical oxidation. Rectangular shape of the CV curves was generally maintained and redox peaks appeared at near $0.4 \mathrm{~V}$ indicating that Faradaic process is now involved due to the oxygenated groups on the CNTs (Figure 3a). ${ }^{23}$ The CV shape was still maintained at the scan rate of $1 \mathrm{~V} / \mathrm{s}$, but the capacitance was significantly reduced at $10 \mathrm{~V} / \mathrm{s}$ unlike the as-grown CNTs. Galvanostatic charge/discharge curve and Nyquist plot indicate that ESR was on the same order of magnitude as that of as-grown CNTs (Figure $3 \mathrm{~b}$ and $\mathrm{c}$ ). A small semicircle in the inset of Figure 3c indicates introduction of Faradaic process. ${ }^{24}$ Figure $3 \mathrm{~d}$ shows how specific capacitance changes as the current density increases. Retention of the specific capacitance was $57 \%$ at 145 $\mathrm{F} / \mathrm{g}$, which is significantly lower compared to that of as-grown CNTs ( 94\%). These results indicate that electrochemical oxidation of the CNTs can enhance the specific capacitance and the specific energy but also degrade the rate performance of the supercapacitors.

Finally, we demonstrate that the specific energy can be improved by using organic solution. The range of operating potential could be wider in organic solution than in aqueous solution. Figure $4 \mathrm{a}$ shows CV curves of as-grown and oxidized
CNTs measured in $1 \mathrm{M} \mathrm{Et}_{4} \mathrm{NBF}_{4} \mathrm{PC}$ solution. Applied potential range was from -1.25 to $1.25 \mathrm{~V}$. CV characteristics such as specific capacitance and sweep rate dependence were comparable to or slightly degraded in organic solution compared to those observed in aqueous solution. This could be mainly due to the increased ESR in organic solution caused by lower mobility of organic electrolytes. ${ }^{25}$ Impedance spectroscopy data are presented in Figure 4b. Specific capacitance was increased by electrochemical oxidation in organic solution as well (Figure 4c). Retention characteristics in organic solution also showed similar trend to those in aqueous solution; specific capacitance of oxidized CNTs reduced more rapidly than that of as-grown CNTs (Figure 4d). Specific power and specific energy of as-grown and oxidized CNTs measured in aqueous and organic solution were summarized in Figure 5. In spite of slight degradation of power characteristics in organic solution due to increased ESR, energy characteristics were improved because of wide operation voltage range. Specific energy and power of as-grown CNTs in organic solution measured at the current density of $30 \mathrm{~A} / \mathrm{g}$ were $8 \mathrm{Wh} / \mathrm{kg}$ and $33 \mathrm{~kW} / \mathrm{kg}$, respectively. Maximum specific energy attainable using oxidized CNTs was $\sim 18 \mathrm{Wh} / \mathrm{kg}$. 
(a)

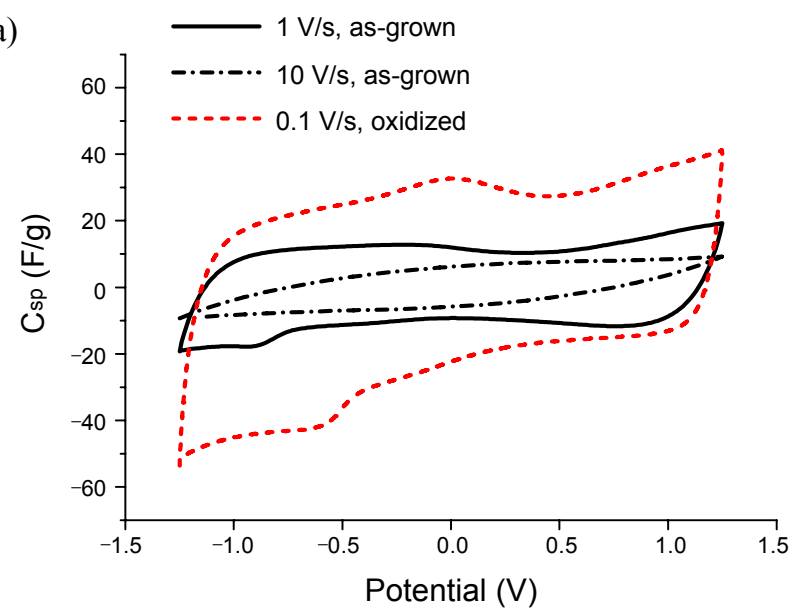

(c)

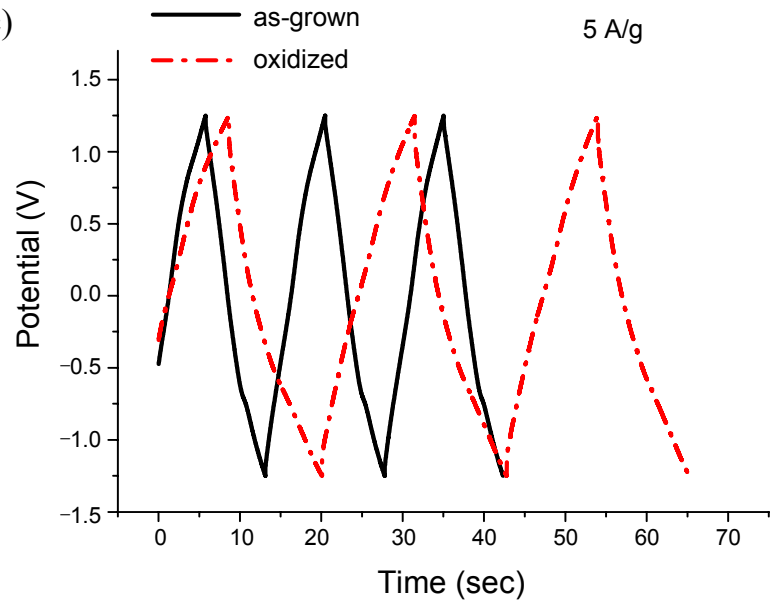

(b)

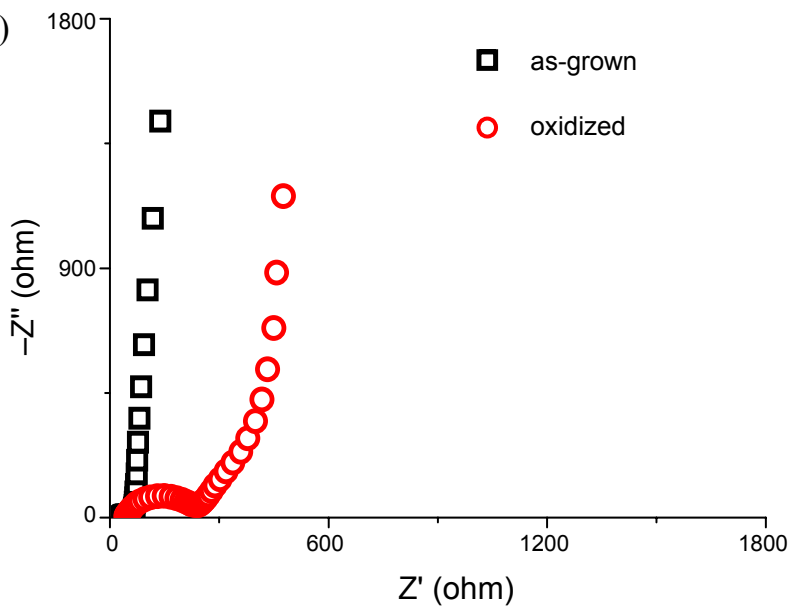

(d)

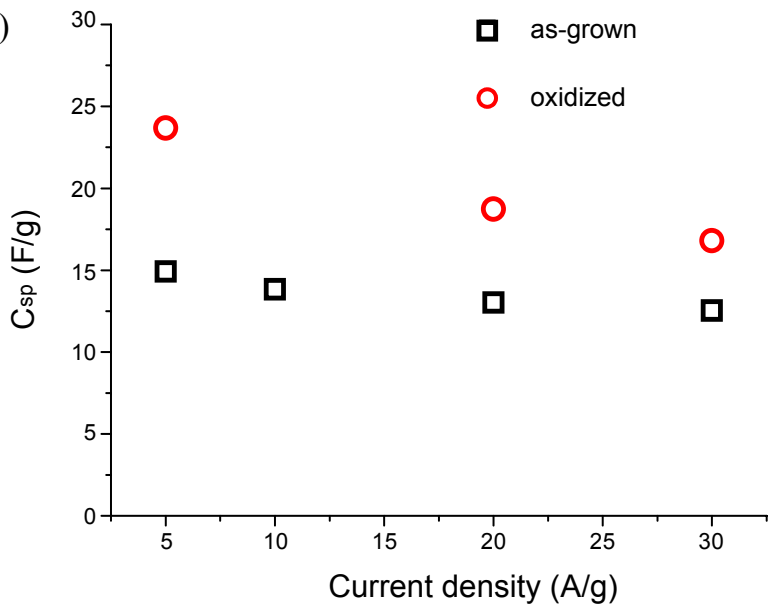

Figure 4. Cyclic voltammograms of (a) as-grown and oxidized CNTs measured in $1 \mathrm{M}$ tetraethylammonium tetrafluoroborate $\left(\mathrm{Et}_{4} \mathrm{NBF}_{4}\right)$ propylene carbonate (PC) solution (b) Nyquist plot. (c) Galvanostatic charge/discharge curves. (d) A plot of specific capacitance vs. current density.

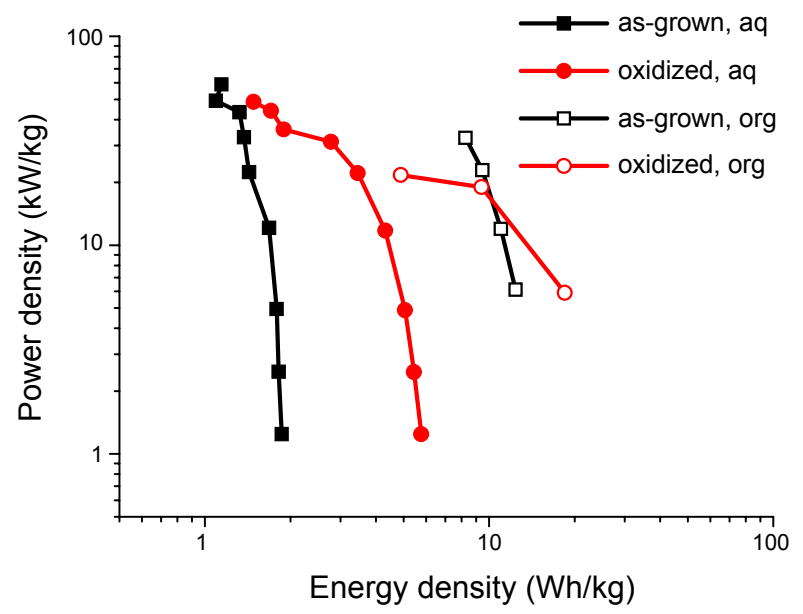

Figure 5. A Ragone plot of as-grown and oxidized CNTs both in aqueous (aq) and organic (org) solution.

\section{Conclusions}

We demonstrate that aligned CNTs can be synthesized directly on Ta substrates via a simple water-assisted CVD process, and that the CNTs can be used as decent electrochemical capacitors. Majority of the CNTs had double walls and had a mean diameter of $\sim 7 \mathrm{~nm}$. Owing to the aligned structure of the CNTs and direct integration with Ta substrates, they showed excellent rate performance; rectangular $\mathrm{CV}$ shapes were observed at fast scan rate $(>1 \mathrm{~V} / \mathrm{s})$ and specific capacitance was mostly retained ( $\sim 94 \%$ ) even when discharge current density was increased up to $\sim 145 \mathrm{~A} / \mathrm{g}$. Specific capacitance and specific energy were improved by electrochemical oxidation of CNTs. Specific energy was further improved in organic solution due to a wide window of operation voltage. However, specific power was slightly degraded due to increased ESR in organic solution. Specific energy and power of the oxidized CNTs in organic solution was 8 $\mathrm{Wh} / \mathrm{kg}$ and $33 \mathrm{~kW} / \mathrm{kg}$, respectively. Further exploration of CNT synthesis directly on diverse current collectors may lead to better understanding of the interface between CNTs and current collectors, and eventually to enhanced supercapacitor performance.

Acknowledgments. This work was supported by the Korea Research Council of Fundamental Science \& Technology (KR$\mathrm{CF}$ ) and Korea Institute of Science \& Technology (KIST) for "National Agenda Project" program, and by the Pioneer Re- 
search Center Program through the National Research Foundation of Korea (No. 2010-0002191).

\section{References}

1. Simon, P.; Gogotsi, Y. Nat. Mater. 2008, 7, 845.

2. Miller, J. R.; Simon, P. Science 2008, 321, 651.

3. Pandolfo, A. G.; Hollenkamp, A. F. J. Power Sources 2006, 157, 11.

4. Kotz, R.; Carlen, M. Electrochim. Acta 2000, 45, 2483.

5. Pan, H.; Li, J. Y.; Feng, Y. P. Nanoscale Res. Lett. 2010, 5, 654.

6. Korenblit, Y.; Rose, M.; Kockrick, E.; Borchardt, L.; Kvit, A.; Kaskel, S.; Yushin, G. ACS Nano 2010, 4, 1337.

7. Shah, R.; Zhang, X. F.; Talapatra, S. Nanotechnol. 2009, 20, 395202

8. Zhang, H.; Cao, G. P.; Yang, Y. S. J. Power Sources 2007, 172, 476.

9. Merkoci, A.; Pumera, M.; Llopis, X.; Perez, B.; Valle, M. del; Alegret, S. Trends Anal. Chem. 2005, 24, 826.

10. Kim, W.; Javey, A.; Tu, R.; Cao, J.; Wang, Q.; Dai, H. J. Appl. Phys. Lett. 2005, 87, 173101

11. Kim, W.; Choi, H. C.; Shim, M.; Li, Y. M.; Wang, D. W.; Dai, H. J. Nano Lett. 2002, 2, 703.

12. Kim, H. S.; Kim, B.; Lee, B.; Chung, H.; Lee, C. J.; Yoon, H. G.; Kim, W. J. Phys. Chem. C 2009, 113, 17983.

13. Zhang, H.; Cao, G. P.; Wang, Z. Y.; Yang, Y. S.; Gu, Z. N. Carbon
2008, 46, 822 .

14. Hiraoka, T.; Yamada, T.; Hata, K.; Futaba, D. N.; Kurachi, H.; Uemura, S.; Yumura, M.; Iijima, S. J. Am. Chem. Soc. 2006, 128 , 13338.

15. Jung, Y. J.; Wei, B. Q.; Vajtai, R.; Ajayan, P. M. Nano Lett. 2003, 3,561 .

16. Talapatra, S.; Kar, S.; Pal, S. K.; Vajtai, R.; Ci, L.; Victor, P.; Shaijumon, M. M.; Kaur, S.; Nalamasu, O.; Ajayan, P. M. Nat. Nanotechnol. 2006, 1, 112 .

17. Kim, B.; Chung, H.; Chu, K. S.; Yoon, H. G.; Lee, C. J.; Kim, W. Synth. Met. 2010, 160, 584.

18. Kim, B.; Chung, H.; Kim, W. J. Phys. Chem. C 2010, 114, 15223.

19. Ye, H. S.; Liu, X.; Cui, H. F.; Zhang, W. D.; Sheu, F. S.; Lim, T. M. Electrochem. Commun. 2005, 7, 249.

20. Hata, K.; Futaba, D. N.; Mizuno, K.; Namai, T.; Yumura, M.; Iijima, S. Science 2004, 306, 1362.

21. Qu, L.; Dai, L. J. Mater. Chem. 2007, 17, 3401.

22. Zhang, H.; Cao, G. P.; Yang, Y. S.; Gu, Z. N. J. Electrochem. Soc. 2008, 155, K19.

23. Andreas, H. A.; Conway, B. E. Electrochim. Acta 2006, 51, 6510.

24. Conway, B. E. Electrochemical Supercapacitors-Scientific Fundamentals and Technological Application; Kluwer Academic: New York, 1999

25. Lewandowski, A.; Olejniczak, A.; Galinski, M.; Stepniak, I. $J$. Power Sources 2010, 195, 5814. 